\title{
ADAPTACIÓN DEL MODELO ESPAÑOL DE GESTIÓN EN TRASPLANTE PARA LA MEJORA EN LA NEGATIVA FAMILIAR Y MANTENIMIENTO DEL DONANTE POTENCIAL
}

\author{
Neide da Silva Knihs', Janine Schirmer², Bartira de Aguiar Roza
}

\begin{abstract}
${ }^{1}$ Doutoranda em Enfermagem pela Escola Paulista de Enfermagem da Universidade Federal de São Paulo (UNIFESP). Professora da Universidade para o Desenvolvimento do Alto Vale do Itajaí. Santa Catarina, Brasil. E-mail: neide@unidavi.edu.br

${ }^{2}$ Doutora em Enfermagem. Professora Titular da Escola Paulista de Enfermagem da UNIFESP. São Paulo, Brasil. E-mail: schirmer.janine@unifesp.br

${ }^{3}$ Doutora em Enfermagem. Professora Adjunta da Escola Paulista de Enfermagem da UNIFESP. São Paulo, Brasil. E-mail: bartira.roza@unifesp.br
\end{abstract}

RESUMEN: El propósito del estudio fue comparar los datos relacionados a la formación y perfil de los coordinadores de trasplante de Brasil y España para evaluar oportunidades de mejoría en la negativa familiar y el mantenimiento del donante en nuestro país. Una minuciosa evaluación fue realizada, comparando los datos de formación del coordinador de trasplante, perfil del coordinador de trasplante y de la metodología de trasplante de cada país. En Brasil el promedio de horas de capacitación recibida antes de empezar a trabajar con trasplantes es de ocho horas, en España de 42 horas. En Brasil 22,9\% de los coordinadores de trasplante son médicos y en España 95\% son médicos. El tiempo que actúa el coordinador de trasplantes en Brasil es de nueve meses y 22 días y en España 60 meses. El Modelo de Gestión de la Organización Nacional de Trasplante, puede ser adaptado en Brasil, como una forma de mejoría en la formación del coordinador de trasplante y de cambiar las tasas por negativa familiar y por pérdidas de mantenimiento.

DESCRIPTORES: Donación de órganos. Trasplante. Habilitación profesional. Enfermería.

\section{ADAPTING THE SPANISH TRANSPLANT MANAGEMENT MODEL TO REDUCE FAMILY REJECTION AND MAINTAIN THE POTENTIAL DONOR}

\begin{abstract}
The purpose of this study was to compare data related to professional education and profiles of transplant coordinators in Brazil and Spain in order to evaluate opportunities for improvement, due to family rejection and maintaining the potential donor within the country. A detailed evaluation was carried out which compares the formal education data of the transplant coordinator, the coordinator's profile, and the transplant methodology of each country. In Brazil, there is an average of eight hours in training prior to beginning work with transplants, whereas in Spain, 42 hours are necessary. In Brazil $22.9 \%$ of transplant coordinators are physicians, while in Spain 95\% are physicians. The average length of time that the transplant coordinator has worked in Brazil was nine months and twenty-two days, while in Spain it was sixty months. The National Transplant Organization Management Model (El Modelo de Gestión de la Organizacion Nacional) may be adapted in Brazil as a way to improve transplant coordinator education and family rejection and maintenance losses.
\end{abstract}

DESCRIPTORS: Organ donation. Transplantation. Credentialing. Nursing

\section{ADAPTAÇÃO DO MODELO ESPANHOL DE GESTÃO EM TRANSPLANTES PARA A MELHORA DA NEGATIVA FAMILIAR E MANUTENÇÃO DO POTENCIAL DOADOR}

\begin{abstract}
RESUMO: O propósito do estudo foi comparar os dados relacionados à formação e perfil dos coordenadores de transplantes do Brasil e Espanha para avaliar oportunidades de melhorias em relação à recusa familiar e manutenção do potencial doador. Uma minuciosa avaliação foi realizada, comparando os dados de formação do coordenador de transplante, perfil do coordenador de transplantes e da metodologia de transplante em cada país. No Brasil, a média de horas de capacitação recebida antes de começar a trabalhar foi de oito horas e na Espanha foi de 42 horas. No Brasil, 22,9\% dos coordenadores de transplante são médicos, na Espanha 95\% são médicos. O tempo que atua o coordenador de transplante no Brasil foi nove meses e 22 dias, na Espanha, 60 meses. O Modelo de Gestão da Organização Nacional de Transplante pode ser adaptado no Brasil como forma de melhoria nas perdas por negativa familiar e por perdas na manutenção.
\end{abstract}

DESCRITORES: Doação de órgãos. Transplante. Qualificação profissional. Enfermagem 


\section{INTRODUCCIÓN}

Los trasplantes de órganos han evolucionado de manera significativa. Este avance ha traído numerosos beneficios a los trasplantes de órganos y junto a esto, importantes dilemas éticos. En el proceso de donación de órganos, los profesionales de la salud cuidan del donante potencial en la expectativa de generar vida a través de la donación.

El profesional que trabaja en la donación de órganos y tejidos, tiene que tener un perfil con numerosas características, entre esas, la importancia de manejarse muy bien con la muerte para que así, se pueda estar junto a la familia y también haciendo el mantenimiento del donante. Esto necesita del conocimiento de todo el proceso, logística, legislación, muerte encefálica: concepto y definición y diagnóstico clínico. ${ }^{1-3}$ El conocimiento no es solamente científico, sino que incluye conocer fisiología, anatomía, leyes, bioética, habilidad para mantener al donante potencial y como un toque especial el cuidado a la familia que se encuentra sufriendo. Así se puede decir que la persona que trabaja en la donación de órganos y tejidos debe tener mucho conocimiento, pero más que esto, hay que tener mucho amor y pasión por lo que hace. . $^{4-6}$

Delante de la aceptación de la muerte por parte de la familia, los profesionales de la salud tienen un gran desafío, el mantenimiento del donante potencial. Después de establecida la Muerte Encefálica (ME), se producen una serie de alteraciones fisiopatologías que conducen a la parada cardiaca si no se realiza un tratamiento adecuado. Todas estas alteraciones convierten al donante en un paciente con muchos cuidados, estos cuidados tienen como propósito una estabilidad hemodinámica con una oxigenación adecuada para todos los órganos y tejidos para que se pueda hacer lo trasplante en las mejores condiciones posibles y así no haga ninguno daño a lo receptor de los órganos. ${ }^{7-8}$

En Santa Catarina y Brasil, así como en la mayoría de los países latinoamericanos, existen algunos problemas relacionados con la donación de órganos. En Brasil los problemas están relacionados con factores como: la subnotificación, problemas en el mantenimiento del donante potencial, negativa familiar y problemas logísticos que están presentes en toda la extensión territorial. En Santa Catarina en los últimos cinco años se ha evaluado de manera muy significativa en la tentativa de cambiar las tasas de donación. El incremento de la donación obtenido se produce por un planteamiento estratégico, el desarrollo de estas actividades, cambió una tasa de donación de 7.3 (pmp) en 2004 por una tasa de 19.8 (pmp) en 2009. Sin embargo, continúa presentando problemas relacionados con el mantenimiento del donante en potencial y negativa familiar lo que significa una pérdida de $88,3 \%$ de los donantes potenciales. ${ }^{9-11}$ La España en los últimos años tiene se destacado en la donación de órganos y tejidos, saliendo de una tasa de 14,4 donantes efectivos (pmp) por año en 1998 para una tasa de 34,2 (pmp) en 2009. Las perdidas por recusa familiar fueran de $13,2 \%$ y $3,2 \%$ por mantenimiento. Convirtiendo este país en una oportunidad de ayuda para otros países. ${ }^{12-14}$ Así justifica-se la necesidad y la oportunidad de identificar puntos de mejorar junto a la Organización Nacional de Trasplante da España para que ceja posible cambiar las altas tasas de pérdidas de potenciales donantes en Santa Catarina y Brasil.

\section{OBJETIVOS}

Comparar los datos relacionados a formación y perfil de los coordinadores de trasplante de Brasil, Santa Catarina y España para evaluar las oportunidades de mejora en relación a la negativa familiar y el mantenimiento del donante. Evaluar la adaptación del modelo y de la metodología de formación de los coordinadores de trasplante, para así proponer acciones posibles de implantación, para la mejora de las tasas de negativa familiar y mantenimiento del donante potencial.

\section{MATERIAL Y MÉTODOS}

Trata-se de un estudio cuantitativo, descriptivo de los datos obtenidos de Brasil, Santa Catarina y España relacionados con la formación y perfil de los coordinadores de trasplante, se ha hecho una comparación para evaluar las oportunidades y así elaborar una propuesta de acciones posibles de implantar para obtener la mejoría en la tasa de la negativa familiar y el mantenimiento del donante.

Los datos de la formación y perfil del coordinador de trasplante de Brasil fueron obtenidos junto al Departamento de trasplante del Hospital Israelita Albert Einstein, de São Paulo. Los referidos datos, fueron recogidos en un Encuentro de Coordinadores de Trasplante en el año 2009, realizado por el hospital citado, denominado como: II Encuentro de las Comisiones Hospitaleras de Trasplantes de Brasil (II ENCIHDOTT). En lo encuentro estaban presentes 158 coordinadores de trasplantes, los datos fueran obtenidos cuando empezó lo curso a través de preguntas hechas 
simultáneamente por medio electrónico y las respuestas ocurran luego después de las preguntas también por medio electrónico. ${ }^{15}$ Los datos de la formación y perfil del coordinador de trasplante de Santa Catarina fueron obtenidos de un trabajo de conclusión del curso de Especialización en donación de órganos y tejidos. ${ }^{16}$ Los datos de los profesionales de España fueran obtenidos de la Organización Nacional de Trasplante (ONT). ${ }^{12}$

Los datos de Brasil y Santa Catarina sobre las tasas de donación, pérdida por mantenimiento y negativa familiar fueron obtenidos a través del Registro Brasileño de Trasplantes (RBT) y de España, de la Organización Nacional de Trasplante (ONT) ${ }^{11-12}$ Después de la obtención de los datos, la autora ha realizado, una minuciosa evaluación de las informaciones y así comparando los datos y la metodología del proceso de trasplante de cada país, identificando las diferencias entre las estructuras, funciones de los coordinadores, calificación y perfil de los coordinadores de trasplante para empezar a trabajar en el proceso de trasplante conforme la legislación. También fueron comparados los datos referentes a la negativa familiar, pérdida por mantenimiento del donante, formación profesional y tiempo de actuación como coordinador hospitalario entre Brasil, Santa Catarina y España. Después de evaluar los datos, los mismos fueron presentados en forma de cuadro, para que así se permitiese una mejor observación de las diferencias identificadas y de los resultados de la evaluación. Este estudio fue previamente aprobado por el Comité de Ética da Universidad Federal de São Paulo, sobe lo número de lo parecer 0964/10 y sigue la resolución 196.

\section{RESULTADOS Y DISCUSIÓN}

En 2009 en Brasil hubo 6.490 notificaciones del donante potencial (34.2 pmp/año) y solamente 1.658 donantes fueron efectuados (8,7pmp/año), hubo 4.832 pérdidas, $45,6 \%$ de estas pérdidas corresponden a la negativa familiar, 20,8\% corresponden a la pérdida por mantenimiento del donante potencial. En España 34,2 (pmp/año) fue efectuado, hubo $13,2 \%$ de pérdida por negativa familiar y $3,2 \%$ de perdidas por mantenimiento del donante potencial. Los datos presentan que el promedio de capacitación recibido en Brasil es de ocho horas, Santa Catarina 12,8 horas y España 42 horas. Con relación a la formación profesional del coordinador de trasplante en Brasil 22,9\% son médicos, Santa Catarina 4,87\% son médicos y España $95 \%$ de estos profesionales son médicos. En relación al tiempo que actúa como coordinador de trasplantes en Brasil nueve meses y 22días, Santa Catarina 12 meses y 23 días y España 60 meses. ${ }^{9-12}$

En la comparación de los datos relacionados a la formación y perfil de los coordinadores de trasplante de Brasil, Santa Catarina y España, se observan algunos puntos del Modelo de Gestión Español que pueden ser muy importantes para establecer un plan de actividades que podría mejorar las tasas de pérdidas por mantenimiento y por negativa familiar en Santa Catarina. Entre los datos y las informaciones evaluados en algunos son más visibles las diferencias, de estos podemos citar el modelo de Coordinación Hospitalaria, la calificación del coordinador, el perfil del coordinador y el tiempo en que el coordinador permanece actuando en este proceso.

\section{Coordinación hospitalaria}

En Brasil, los coordinadores de trasplante son personas de los propios hospitales, la legislación determina que todos los hospitales que tienen paciente en ventilación mecánica en Unidad de Crítico deben tener coordinador de trasplante. ${ }^{15}$ En la práctica hay una gran diferencia en todo lo territorio brasileño, en la región Sur hay coordinadores en casi todos los hospitales, en la región Sudeste en especial São Paulo hay Organización de Procura de Órganos (OPO) y en otros estados de la región Norte y Nordeste las centrales estaduales coordinan la mayoría de los procesos..$^{17}$ A pesar de existir desde hace cinco años una legislación, ${ }^{18-19}$ a cual determina la creación de los equipos de coordinación, en la mayoría de los estados no hay coordinador en todos los hospitales.

En Santa Catarina hay 38 hospitales con ventilación mecánica y 17 hospitales con neurocirugía. De los hospitales con neurocirugía en todos hay coordinación, sin embargo, no son todos los que hacen notificaciones de ME, solamente nueve hacen las notificaciones de ME con frecuencia, cinco de estos son los hospitales que hacen $70 \%$ de las notificaciones del estado. ${ }^{10}$

Cuando se evalúan los datos de España se puede observar que la coordinación está hecha por los profesionales de los propios hospitales y en todo el territorio se hace de la misma manera, en todos los hospitales que hay Unidad de Paciente Crítico (UCI), hay coordinación, notificación de ME y donantes reales.

Los equipos de coordinación están formados por médicos y enfermeros, pero 95\% de los coor- 
dinadores de España son médicos. También se da prioridad que sea médico de la institución, por la facilidad que hay en discutir casos más difíciles con otros médicos; de preferencia intensivistas, que están donde hay donantes; y equipo restante de acuerdo con el tipo de hospital (mayor número si tiene servicios de trasplante y neurocirugía), y nunca subordinado a algún equipo de trasplante, para evitar cuestiones éticas. ${ }^{3}$

\section{Calificación del coordinador de trasplante}

La legislación de Brasil, válida para todo el territorio nacional determina que el coordinador hospitalario deberá participar del Curso de formación de coordinadores, con la carga horaria de un mínimo de 24 hs, antes de asumir la coordinación. El curso tendrá los siguientes contenidos: Detección del donante, selección del donante, mantenimiento del donante, diagnóstico de ME, entrevista familiar para donación de órganos, extracción de órganos y tejidos, logística del proceso de donación, ética en donación de órganos, criterios de distribución de órganos y aspectos legales. ${ }^{19}$

La formación de médicos y enfermeras ha sido y sigue siendo uno de los pilares fundamentales sobre los que se asienta el modelo español de donación y trasplantes. La metodología docente utilizada por el Programa de Formación de Coordinadores, aborda el método de enseñanza interactivo y participativo, basado en el modelo de aprendizaje por experiencias.

En España antes de que el coordinador empiece sus actividades, este hace un curso general de donación de órganos y tejidos basado en tópicos principales como: detección del donante, diagnóstico de $\mathrm{ME}$, mantenimiento del donante, curso comunicación de malas noticias, entrevista familiar para donación, criterios de distribución de los órganos. Junto a las clases teóricas se hacen clases prácticas en centros preparados como si fuese la propia realidad (llamadas talleres). Además se realizan cursos para intensivistas, profesionales que actúan en las unidades de emergencias y reanimación y también se hacen dos cursos por año para todos los profesionales del hospital y así concientizar sobre donación de órganos. ${ }^{20}$

El modelo español trata de solucionar el aumento de la demanda de órganos para trasplante, con la actuación de los profesionales en la detección del donante y el mantenimiento del donante como puntos estratégicos. Condiciona que el intensivista no termine su trabajo al realizar el diagnóstico de ME, sino que desde ese momento, y cambiando el esquema terapéutico, sus esfuerzos deben dirigirse a mantener el "cadáver latiente" en condiciones óptimas de perfusión y oxigenación para tratar de mejorar la viabilidad de los órganos que van ser posteriormente implantados. ${ }^{21-22}$.

En el modelo español los profesionales de la salud que actúan junto a los donantes deben estar preparados para tratar las alteraciones fisiológicas de todos los sistemas orgánicos, envolviendo alteraciones bioquímicas y celulares que conducen a disfunción multiorgánica cuando ocurre la ME. En la metodología de España los profesionales que actúan en unidades que tengan donantes potenciales están preparados para realizar el mantenimiento del donante ante todas estas alteraciones hemodinámicas, aunque estos no sean coordinadores. ${ }^{21-22}$

Para los profesionales de la salud es un gran desafío, conseguir la estabilidad hemodinámica del donante y conseguir que los órganos que van a ser trasplantados estén en buenas condiciones de perfusión. El mantenimiento del donante consiste en medidas terapéuticas que deben ser tomadas para asegurar la estabilidad hemodinámica. Pero para que esto ocurra se hace necesario que sean entrenados, este es uno de los factores deficitarios en Brasil y Santa Catarina. Cuando evaluamos los datos del entrenamiento de los coordinadores de trasplantes en Brasil la carga horaria fue de ocho horas y en Santa Catarina de 12.8 hs, esto demostró que la carga horaria está muy por debajo de lo que determina la legislación brasileña.

Otro punto muy enfocado en el entrenamiento del modelo Español es la preparación de los profesionales para la atención a la familia del donante. La preparación de las personas que actúan en las unidades donde hay donantes consiste en entrenarlos para comunicar la mala noticia y la entrevista para donación. La acogida y la entrevista familiar, son consideradas parte del bagaje histórico de los avances técnicos que han permitido el espectacular auge de los trasplantes. ${ }^{5,20}$

La falta de acogida, la incertidumbre, la falta de atención a la familia en este proceso se reveló como una falta de estímulo para la autorización de la donación. La baja calidad de las informaciones, la dificultad de acceso y las contradicciones produjeron una sensación de abandono por los familiares. ${ }^{23-24}$

El equipo de salud implicado en el proceso de donación, generalmente está formado por profesionales con significativo/importante conocimiento técnico y científico. Sin embargo, con 
notables dificultades y dilemas para comunicar una mala noticia junto al paciente y su familia.

Nosotros, profesionales de la salud, estamos preparados para actuar de forma hábil para salvar vidas. Todavía hay mucho desfase en la academia, en cuanto a la preparación de los profesionales para estar junto al paciente y la familia en el momento de comunicar una mala noticia. La mala noticia está marcada por eventos que involucran el comunicar al paciente/familia sobre el agravamiento del cuadro clínico, diagnóstico donde no hay perspectivas de mejora, estado terminal, cuidados paliativos, fin de vida y comunicado de óbito. ${ }^{25}$

Los profesionales sanitarios debemos ayudar a estas familias a aceptar y entender la muerte encefálica, de igual manera, que a los familiares de los pacientes que fallecen en asistolia. Se hace necesario que nosotros estemos preparados para conducir ese proceso y explicarle a la familia que muerte sólo hay una, lo que cambia es la forma de llegar a ella. En este momento lo que la familia necesita es la acogida, la escucha y atención de los profesionales. ${ }^{5,25-26}$

En el modelo español se entiende que para que ocurra una identificación eficaz del donante, así como un buen mantenimiento y una adecuada entrevista familiar para donación, se hace necesario primero que los profesionales estén preparados para actuar en este proceso..$^{20-21}$

Es responsabilidad del equipo de salud realizar el mantenimiento y el control de todos los datos hemodinámicas del donante. Por tanto, se hace necesario que el equipo de salud este preparado para identificar el donante potencial, así como hacer el mantenimiento, la acogida y la entrevista para donación. El equipo que actúa en las unidades que acogen los donantes potenciales, así como el coordinador forman gran parte del éxito de los trasplantes. $3,13,20-23$

\section{Perfil del coordinador de trasplante}

En la legislación de Brasil el coordinador podrá ser un médico o un enfermero, depende de la complejidad del hospital. Los datos presentan que $22,9 \%$ de los coordinadores son médicos en Brasil y España 95\% son médicos y de estos 79\% son intensivistas.

En Brasil así como en Santa Catarina la mayoría de los coordinadores son enfermero, sin duda este profesional tiene gran conocimiento para actuar junto al equipo de trasplante, pero al comparar los datos se puede objetivar la gran diferencia en las pérdidas por mantenimiento comparados con España, donde el profesional de coordinación se hace en $79 \%$ por un intensivista.

En el modelo Español los intensivistas son los profesionales que mejor se adaptan para hacer la coordinación de trasplante por estar estos muy cerca del donante y así tienen mayor posibilidad de identificar y hacer el adecuado mantenimiento del donante, por la preparación que este profesional recibió a largo de su vida profesional. Se puede percibir que hay una gran participación de los intensivistas en todo el proceso, aunque este profesional no forme parte de la coordinación; desde la residencia empieza a hacer cursos de donación de órganos, y el proceso de entrenamiento tiene continuidad durante la vida profesional en las UCIs. ${ }^{3,20}$

\section{Tiempo en que el coordinador permanece actuando en este proceso}

En España se dice que el coordinador tiene un tiempo para estar en esta actividad. Hablan del síndrome del coordinador "quemado", porque la actividad del coordinador con el paso de los años se va desgastando por el cansancio físico y emocional. ${ }^{13,20}$ Pero así mismo, el tiempo en la coordinación no es tan corto como ocurre en Brasil y en Santa Catarina. También se puede comentar que en España todos los profesionales que actúan en el proceso de donación son remunerados, desde el médico, enfermero, cirujano y otros.

Se puede percibir que hay una gran diferencia del tiempo de los coordinadores entre Brasil y España. Lo que ocurre en Brasil y Santa Catarina es que los coordinadores no se sienten preparados para actuar en esta actividad, junto a estos la mayoría de estas personas no reciben ningún beneficio por hacer la función de coordinador.

Así, estos profesionales acaban por cambiar con mucha frecuencia, en Santa Catarina el promedio tiempo de un coordinador es solamente de nueve meses y 22 días. A lo largo de la discusión se refleja la importancia del conocimiento de los coordinadores acerca del proceso del trasplante, demostrando la importancia de cambiar esta realidad en Santa Catarina. Las pérdidas y negativas familiares para donación se justifican por las bajas horas de entrenamiento de estos profesionales.

\section{CONCLUSIONES}

El Modelo de Gestión del Proceso de Donación/extracción de Órganos y Tejidos de la ONT, 
España, puede ser adaptado a Santa Catarina y Brasil, como una forma de mejora y de cambiar las pérdidas por negativa familiar y por mantenimiento del donante potencial. Con la evaluación de los datos fue posible identificar puntos significativos en la formación y el perfil profesional del coordinador. Así, para que ocurra esta adaptación y se cambie la realidad en el estado se hace necesario algunas acciones de la Central de Trasplantes de Santa Catarina junto a los hospitales que tienen coordinadores de trasplantes.

\section{Acciones propuestas:}

1. Implantación de un programa de garantía de calidad trasladado del modelo de la Organización Nacional de Trasplantes de España, en dos hospitales estratégicos (como piloto). A través de este programa se hace posible identificar las causas de los motivos de la negativa familiar, al mismo tiempo que es posible presentar a los gestores la importancia financiera que su hospital está perdiendo cuando no hace la notificación y cuando no ocurre la donación. Con estos datos se puede presentar a los gestores cuanto se puede pagar a un coordinador, a través de la demostración de cuanto el hospital podría recibir y así pagar una parte a este profesional.

2. A través de estos datos sensibilizar al equipo de salud (todos los profesionales que actúan junto a los posibles donantes y también a todos los profesionales de la institución de salud) de las pérdidas de donantes y del número de personas que no fueron trasplantadas. Junto a esto, se puede ampliar esta sensibilización de la importancia de donación de órganos a las escuelas, universidad y sociedad.

3. Adecuación del entrenamiento que se esta haciendo en Santa Catarina al modelo utilizado en España, con especial atención a la carga horaria y a los contenidos. Con énfasis en la comunicación de mala noticia, entrevista familiar y mantenimiento del donante donde están los mayores problemas.

4. Participación de un médico intensivista en el equipo de trasplante, si no fuera posible ser el coordinador, que forme parte del equipo auxiliando a los otros profesionales.

5. Implantación de Coordinador en todos los hospitales con ventilación mecánica y estimulación de los equipos para que hagan las notificaciones de potenciales donantes a la Central de Trasplantes de Santa Catarina.

6. Curso de donación de órganos a todos los profesionales que trabajan en unidades de emer- gencias y en unidades donde hagan ventilación mecánica, para que estos se sientan preparados para la identificación del donante, el mantenimiento y la acogida a la familia.

Las estrategias para reducir las pérdidas por mantenimiento y por negativa familiar constituyen un elemento clave para aumentar el número de trasplante de órganos.

\section{REFERENCIAS}

1. Bousso RS. Un tiempo para llorar: la familia dando sentido a la muerte de su hijo [tese livre-docência]. São Paulo (SP): Universidad de São Paulo, Escuela de Enfermería; 2006.

2. Guarino AJ. Stress y donación de órganos: una realidad vivida por los enfermeros [disertación]: São Paulo (SP): Universidad de São Paulo, Escuela de Enfermería; 2005.

3. Romero J. Funciones del coordinador de trasplante. En: IX Curso sobre el proceso de donación y trasplante. Valladolid (ES): Libro de Ponencias; 2008.

4. Roza BA. Los efectos de lo proceso de donación de órganos y tejidos en las familias de donantes: intencionalidad de una nueva donación [tese]. São Paulo (SP): Universidad Federal de São Paulo, Escuela Paulista de Enfermería; 2005

5. Santiago C. La entrevista familiar. En: V Curso Internacional de Coordinación de Trasplante. Granada (ES): Iavante Consejería de Salud; 2010. p. 225-50.

6. Santiago C, Gómez P, Mira S, Pérez D, Fuentes M, Olivares J, et al. Cross-cultural donación and donación Interview. Transplant Proc. 2008 Nov; 40(9):2881-2

7. Perez JM. Mantenimiento general del donante. En: V Curso Internacional de Coordinación de Trasplante. Granada (ES): Iavante Consejería de Salud; 2010 p. 50-75.

8. Guetti NR, Marques IR. Asistencia de enfermería al potencial donante de órganos en morte encefálica. Rev Bras Enferm. 2008 Jan-Fev; 61(1):91-7.

9. Asociación Brasileña de Trasplante de Órganos [página en la internet]. São Paulo (SP): ABTO; 2010 [acceso 2010 Mar 10]. Disponible en: http://abto. com/estatisticas.

10. Central de Trasplantes de Santa Catarina [página en la internet]. Florianópolis (SC): CNCDO; 2010 [acceso 2010 Mar 10] Disponible en: http:// sctransplantes.saude.sc.gov.br/estatísticas

11. Registro Brasileiro de Trasplantes. Estadística 2009 de la Asociación Brasilera de trasplante de órganos [página en la internet]. [acceso 2010 Mar 10]. Disponible en: www.abto.com.br

12. Organización Nacional de Trasplantes [página en la internet]. España: ONT; 2010. [acceso 2010 Mar 20] Disponible en: http://ont.es/especialidad. 
13. Matesanz R. Los inicios. In: Matesanz R. El milagro de los trasplantes. Madrid (ES): La Esfera de los Libros, 2006. p. 15-39.

14. López C. El éxito del modelo español de donación de órganos. La vanguardia [online], 2008 Jan 22 [acesso 2010 Mar 10]; Las fronteras de la medicina. Disponible en: http:/ /www.lavanguardia.com/ vida/20080122/53429055057/el-exito-del-modeloespanol-de-donacion-de-organos.html

15. Sociedad Benefícienle Israelita Brasileira. Hospital Albert Einstein [página en la Internet]. São Paulo: 2010 [acceso 2010 Mar 20]. Disponible en: http:// einstein.br/hospital/trasplante

16. Silva RM, Batista J, Knihs NS. Nivel de conocimiento de los alumno de un curso de pós-grado en donación de órganos que actúan en lo proceso de trasplante. In: Anaïs de Trasplante do XI Congreso Brasileño de Trasplante, 2009 Oct 13-16; Recife, Pernambuco, Brasil. Recife (PE): Ed. Eventos Empresarial; 2010. Oficina 488.

17. Garcia VD. A política de trasplantes en Brasil. AMRIGS. 2006 Out-Dez; 50(4):313-20

18. Brasil. Portaria $\mathrm{n}^{\mathrm{o}} 1.752$, de 23 de setiembre de 2005: determina la creación de las comisiones intra-hospitaliza de donación de órganos y tejidos para trasplante en todo los hospitales públicos, filantrópico y privados. Diario Oficial de la República Federativa de lo Brasil, 23 de setiembre 2005.

19. Brasil. Portaría $n^{\circ} 1262$, de 16 de junio de 2006: regalamiento técnico y establece las atribuciones y los deberes y indicadores de las comisiones intrahospitaliza de donación de órganos y tejidos para trasplante. Diario Oficial de la República Federativa de lo Brasil, 16 de junio de 2006.

20. Robles JC. Funciones de coordinador de trasplante. En: V Curso internacional de coordinación de trasplante. Granada (ES): Iavante Consejería de Salud; 2010. p. 24-40.

21. Mingo PU. Mantenimiento clínico del donante de órganos. En: IX Curso sobre el proceso de donación y trasplante. Valladolid: Libro de Ponencias; 2008.

22. Seller G, Hinofosa R. Mantenimiento del donante de órganos. Med Intensiva. 2009 Jun-Jul; 33(5):90-1.

23. Cuende N, Cuende J, Fajardo JI, Huet J, Alonso M. Effect of population aging on the international organ donation rates and the effectiveness of the donation process. Am J Transplant. 2007; 7:1526-35

24. Dailbert MC. Negativa familiar para donación de órganos, Central de Trasplante, CNCDO Regional Zona da Mata / Minas Gerais [dissertação]. Minas Gerais (MG): Universidade Federal de Juiz de Fora, Faculdade de Serviço Social; 2006.

25. Leal F. Transmisión de malas noticias. In: Curso de Donación Trasplante de Órganos en Medicina Intensiva. Granada, 2003. Rev Port Clin Geral. 2003; 19: 40-3.

26. Bernieri J, Hirdes A. Lo preparo de los académicos de enfermería brasileños para la vivencia del proceso morte-morrer. Texto Contexto Enferm. 2007 JanMar; 16(1):89-96. 